\title{
Modeling escape from a one-dimensional potential well at zero or very low temperatures
}

Cite as: J. Appl. Phys. 127, 143901 (2020); https://doi.org/10.1063/1.5144233

Submitted: 03 January 2020. Accepted: 29 March 2020 . Published Online: 14 April 2020

Chungho Cheng, Gaetano Salina, Niels Grønbech-Jensen, James A. Blackburn, Massimiliano Lucci, and Matteo Cirillo (i)
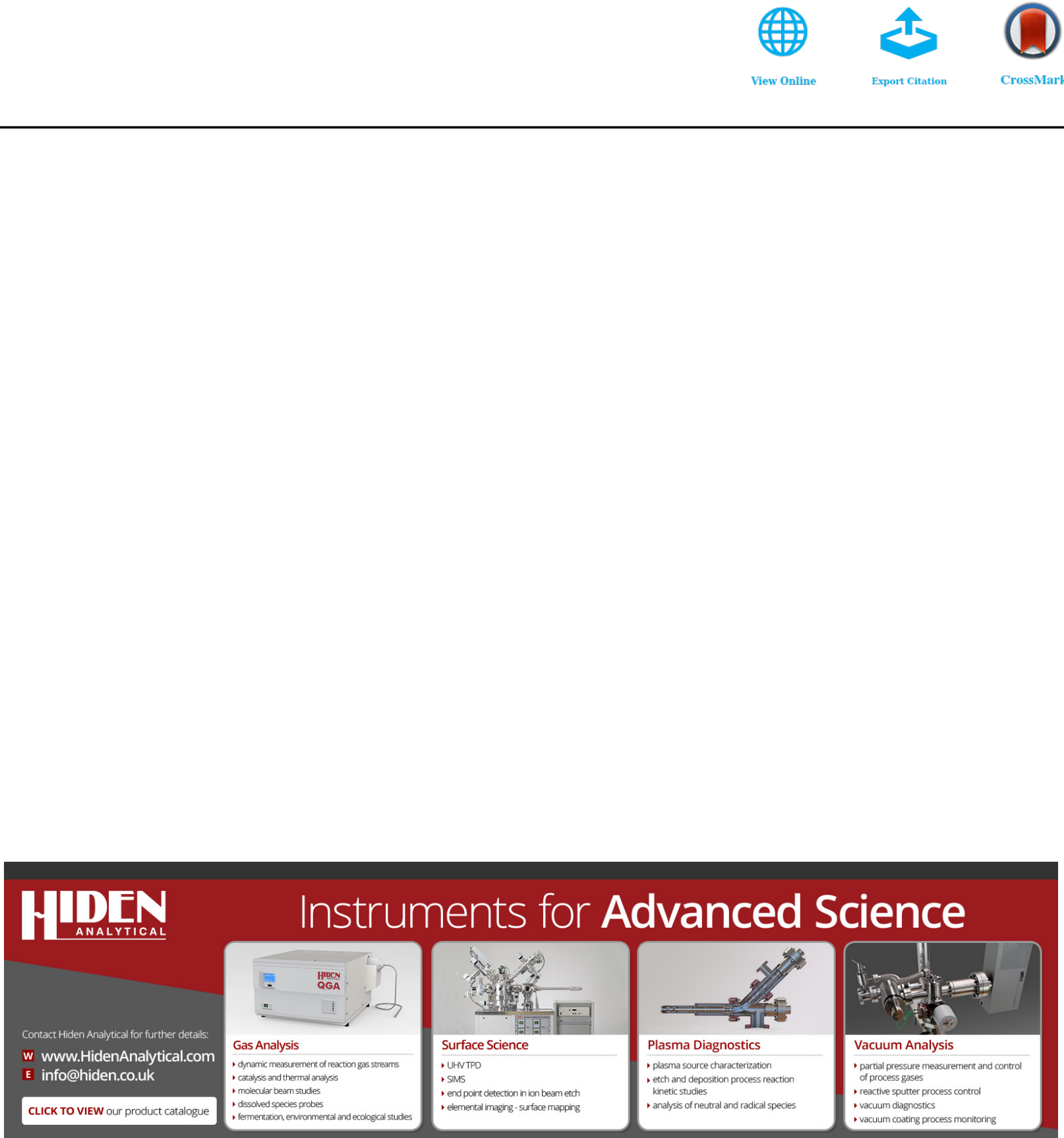


\title{
Modeling escape from a one-dimensional potential well at zero or very low temperatures
}

\author{
Cite as: J. Appl. Phys. 127, 143901 (2020); doi: 10.1063/1.5144233 \\ Submitted: 3 January 2020 . Accepted: 29 March 2020 . \\ Published Online: 14 April 2020
}

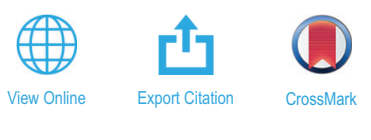

Chungho Cheng, ${ }^{7}$ Gaetano Salina, ${ }^{2}$ Niels Grønbech-Jensen, ${ }^{1,3}$ James A. Blackburn, ${ }^{4}$ Massimiliano Lucci, ${ }^{5}$ and Matteo Cirillo ${ }^{5, a)}$ (iD

\author{
AFFILIATIONS \\ ${ }^{1}$ Department of Mechanical and Aerospace Engineering, University of California, Davis, Davis, California 95616, USA \\ ${ }^{2}$ Istituto Nazionale di Fisica Nucleare, Sezione Roma "Tor Vergata," I-00133 Roma, Italy \\ ${ }^{3}$ Department of Mathematics, University of California, Davis, Davis, California 95616, USA \\ ${ }^{4}$ Physics and Computer Science, Wilfrid Laurier University, Waterloo, Ontario N2L 3C5, Canada \\ ${ }^{5}$ Dipartimento di Fisica and MINAS-Lab, Università di Roma "Tor Vergata," I-00133 Roma, Italy
}

a) Author to whom correspondence should be addressed: cirillo@roma2.infn.it

\begin{abstract}
The process of activation from a one-dimensional potential is systematically investigated in zero and nonzero temperature conditions. The features of the potential are traced through statistical escape from its wells, whose depths are tuned in time by a forcing term. The process is carried out for the damped pendulum system imposing specific initial conditions on the potential variable. While the escape properties can be derived from the standard Kramers theory for relatively high values of the dissipation, for very low dissipation, these deviate from this theory by being dependent on the details of the initial conditions and the time dependence of the forcing term. The observed deviations have regular dependencies on initial conditions, temperature, and loss parameter itself. It is shown that failures of the thermal activation model are originated at low temperatures and very low dissipation, by the initial conditions and intrinsic, namely, $T=0$, characteristic oscillations of the potential-generated dynamical equation.
\end{abstract}

Published under license by AIP Publishing. https://doi.org/10.1063/1.5144233

\section{INTRODUCTION}

The interest for fluctuations in dynamical systems and the analysis of their dynamical response when subject to external forcing terms and/or thermal noise is a subject that has attracted scientists of many generations. Several relevant reviews are available on this topic, ${ }^{1-3}$ and the body of work and insights are still expanding in part due to the increased power of numerical techniques. A key model for describing thermal activation is due to Kramers's; ${ }^{4,5}$ a relevant feature of this model is that the escape rate $\Gamma$ from a potential well with amplitude $\Delta U$, at temperature $T$, is governed by the equation ${ }^{4}$

$$
\Gamma=f \exp \left(-\frac{\Delta U}{k_{B} T}\right),
$$

where $f$ is an attempt frequency. A system to which the Kramers model [and Eq. (1)] has been extensively applied is the compound pendulum, which is described by the following normalized equation for the angle $\varphi$ :

$$
\ddot{\varphi}+\alpha \dot{\varphi}=-\frac{d U}{d \varphi}
$$

with $U(\varphi)=(1-\cos \varphi)-\eta \varphi$ being the potential energy. The coefficient $\alpha$ and the parameter $\eta$ account for dissipation and forcing terms, respectively. Two recent reviews ${ }^{6,7}$ demonstrate the relevance of system (2) in the history of physics and show its intriguing counterparts in general physics and nonlinear dynamics. In condensed matter, the driven-damped pendulum equation is analogous to that of the Josephson junction, ${ }^{8}$ which has been the focus of much attention in theoretical, experimental, and applied physical levels for decades. ${ }^{9,10}$ Specific applications of Kramers analysis to Josephson potentials were reported in two key publications by Kurkijärvi ${ }^{11}$ and Büttiker et al. ${ }^{12}$ 
Due to the applicability of the Kramers model to a broad class of systems, we have conducted a systematic statistical analysis of its validity for describing the behavior of the pendulum. We first study, in Sec. II, the potential in the absence of temperature fluctuations, i.e., studying Eq. (2) in the absence of noise. In this $T=0$ case, we focus on the dependence of the escape from the potential for different dissipation values while increasing the force term linearly on time. We study the effect of specific initial conditions on the statistical distributions of the escape processes analyzing the response induced by the initial data. We also present a model that explains the underlying physics of the obtained numerical results. Then, in Sec. III, we include thermal effects through a noise term in Eq. (2). We show that the conclusions made for the zero temperature case about the initial conditions can play a crucial role for the escape statistics in the regime of low dissipation and nonzero temperature. In Sec. IV, we discuss the results in terms of experimental phenomenology, while in Sec. V, we summarize the paper.

\section{ZERO TEMPERATURE}

In Fig. 1(a), the curves show the first well of the potential $U$ that we are investigating traced for increasing values of the parameter $\eta(\eta=0.6$, the lowest and $\eta=1$ the highest). The inset shows the value of the height of the potential barrier $\Delta U$, the energy spacing between the maximum and minimum value in the well, spanned over the entire interval $[0 ; 1]$ of the forcing term $\eta$; according to the dependence $\Delta U=2\left(\sqrt{1-\eta^{2}}-\eta \cos ^{-1} \eta\right)$, the depth of the potential (the difference in energy between the successive minimum and the maximum) decreases and goes to zero for $\eta=1$ as shown in the inset. In our escape experiments and numerical procedures, the forcing term is increased according to $\eta(t)=\dot{\eta} t$ where the derivative $\dot{\eta}=\frac{d \eta}{d t}$ is kept constant and the curves in Fig. 1(a), in practice, correspond to successive time shots for $\dot{\eta}=1.95 \times 10^{-8}$. In the figure, we also indicate the stable equilibrium points at the bottom of the potential (full diamonds) and the unstable (empty circles) equilibrium points at the top of the well: these were calculated for each curve evaluating numerically the minima and the maxima.

In Fig. 2, we trace both stable and unstable equilibrium points as a function of $\eta$, respectively, by the continuous and the dashed lines: the crossing of the two curves is the point where an escape event is recorded: we see that this event occurs, always when $\eta=1$, when the well becomes flat and the two curves cross, meaning that the stable and unstable equilibrium points have the same $(\eta, \varphi)$

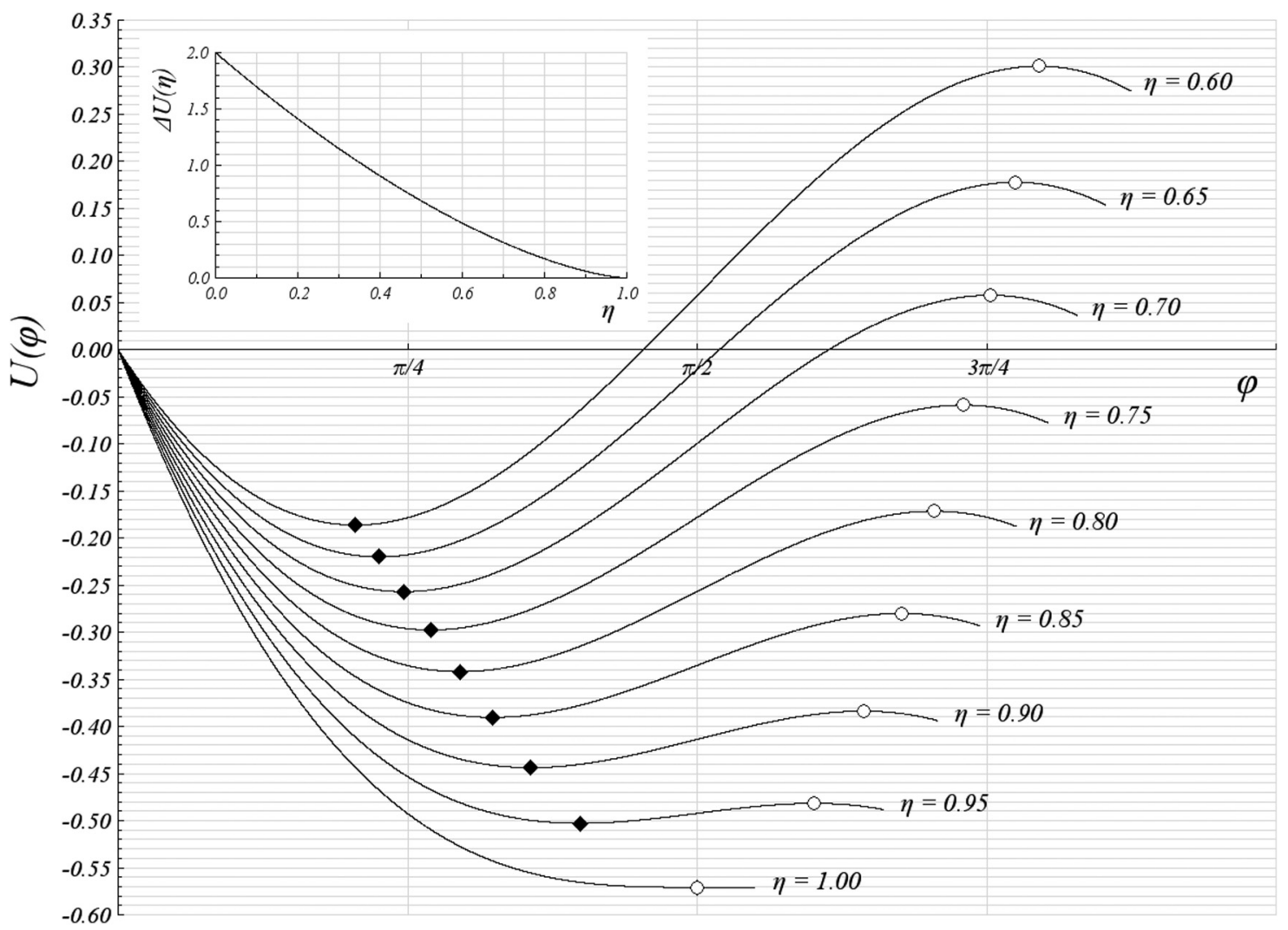

FIG. 1. The process of lowering the potential barrier of system (2) by the external forcing term $\eta$. The inset shows the dependence of the amplitude of the potential (the difference in energy between the maximum and the minimum) as a function of $\eta$. The full diamonds and the empty circles indicate the stable and unstable points of the potential, respectively. Escape from the well occurs when stable and unstable points coincide. 

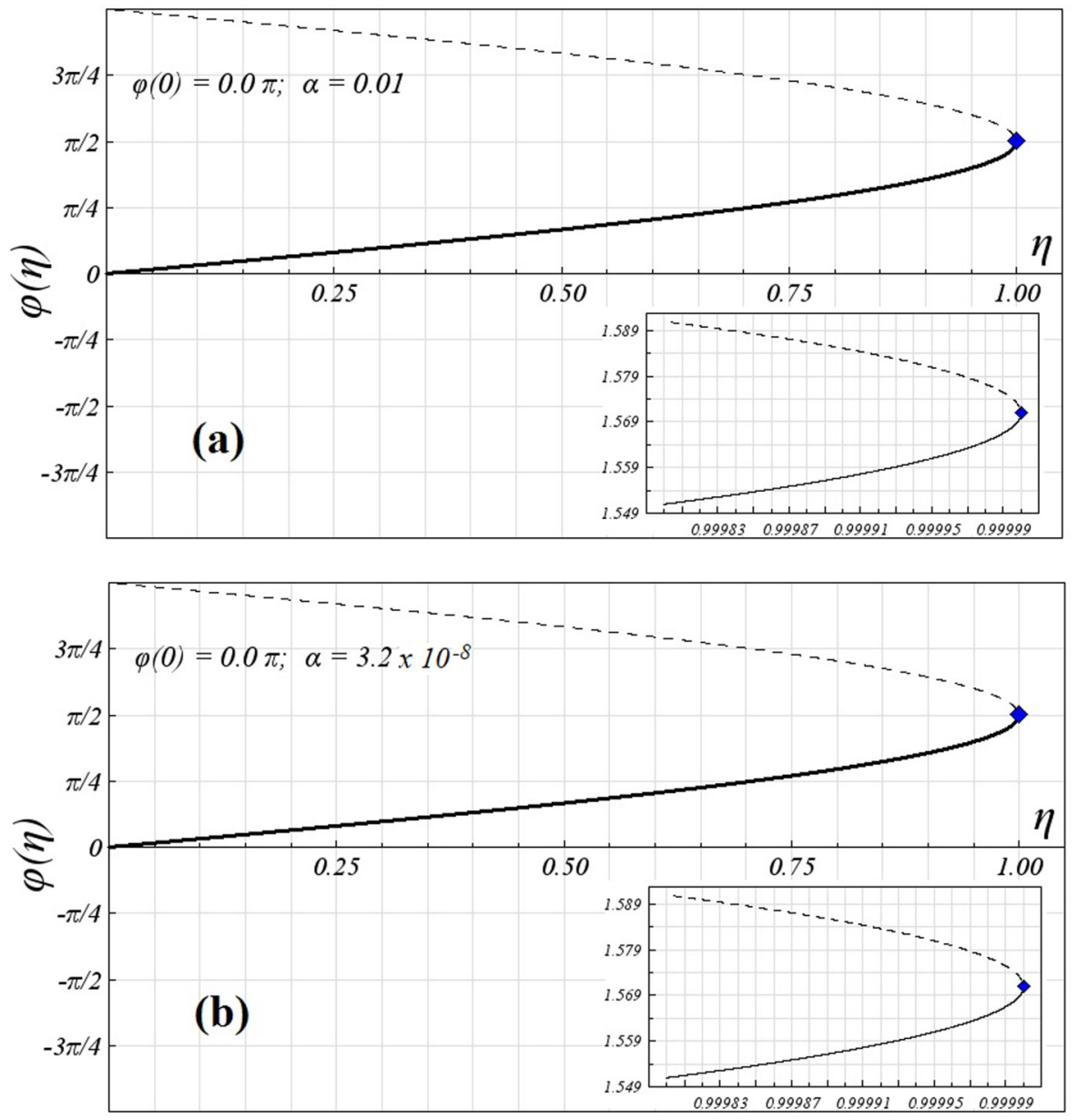

FIG. 2. Traces of the minimum (continuous curve) of maximum (dashed curve) of the well in Fig. 1 traced as a function of $\eta$ for "flat" initial conditions, namely, $\varphi=0$ and $\dot{\varphi}=0$. As indicated in the plots, (a) and (b) correspond to loss factors $\alpha$ that are 6 orders of magnitude apart. Escape occurs when the two curves cross and we see that, in spite of the noticeable difference in loss, it occurs in both cases for $\eta=1$, in this integration. coordinates. The escape event is numerically identified by the fact that, for a further increase of the forcing term above $\eta=1$, the system is driven in a dynamical condition of continuous phase increase (rotation states in terms of the compound pendulum). For $T=0$ (zero temperature) and initial conditions $\varphi_{0}=\varphi(t=0)=0$ and $\dot{\varphi}_{0}=\frac{d \varphi}{d t}(t=0)=0$, the escape from the potential well always occurs for $\eta=1$; moreover, for these initial conditions, this situation remains the same, for any value of $\alpha$ and $\eta$. The two plots in Figs. 2(a) and 2(b) represent indeed the results of the numerical integration for these initial conditions and different values of $\alpha$ (indicated in the plot): we can see that, even decreasing the loss parameter of six orders of magnitude, the response is always the same and the escape always occurs above $\eta=1$.

Before proceeding, however, it is necessary to specify what is our criterion to judge that an escape process out of the potential well has safely occurred. When $\eta<1$, the escape occurs at the angle $\varphi=\pi-\arcsin \eta$, while for $\eta \approx 1$, the escape is recorded when $\varphi=\frac{\pi}{2}$. In both cases, the escape from the well is double checked by the continuous increase of the phase generating a non-zero average value of the time derivative of the phase (we usually stop the integration when $\langle\dot{\varphi}\rangle=1$ ). The results herein presented have been obtained integrating the system (2) by specific versions of the velocity-explicit Størmer-Verlet algorithm ${ }^{13}$ setting the integration time $\Delta t=0.02$; two independently developed versions of the algorithm, running on different computers and operating systems, always returned the same results. Halving tests for the integration time step were performed routinely in order to check the independence of the results upon it.

Keeping the same value of $\dot{\eta}=1.95 \times 10^{-8}$ but setting nonzero values for the initial angle $\varphi_{0}$, the dependence on the loss parameter of the escape process becomes significant and the values of $\eta$ for which escape from the potential well are recorded can be substantially different. Figures 3 and 4 show two escape processes obtained both for initial condition $\varphi_{0} \neq 0$ (respectively $\varphi_{0}=0.3 \pi$ and $\varphi_{0}=0.6 \pi$ for Figs. 3 and 4 ) and three decreasing values of the loss parameter $\alpha$. The value of time derivative of the initial phase $\dot{\varphi}_{0}$ was set to zero. The physical meaning of these initial conditions for the system (2) corresponds to start an integration in an oscillating regime with zero average phase derivative in the point where the phase has its maximum value and the derivative of the phase is zero, namely, the inversion point of the oscillations. We see now that in Figs. 3(a), 3(b), 4(a), and 4(b), the "phase trajectory" still 

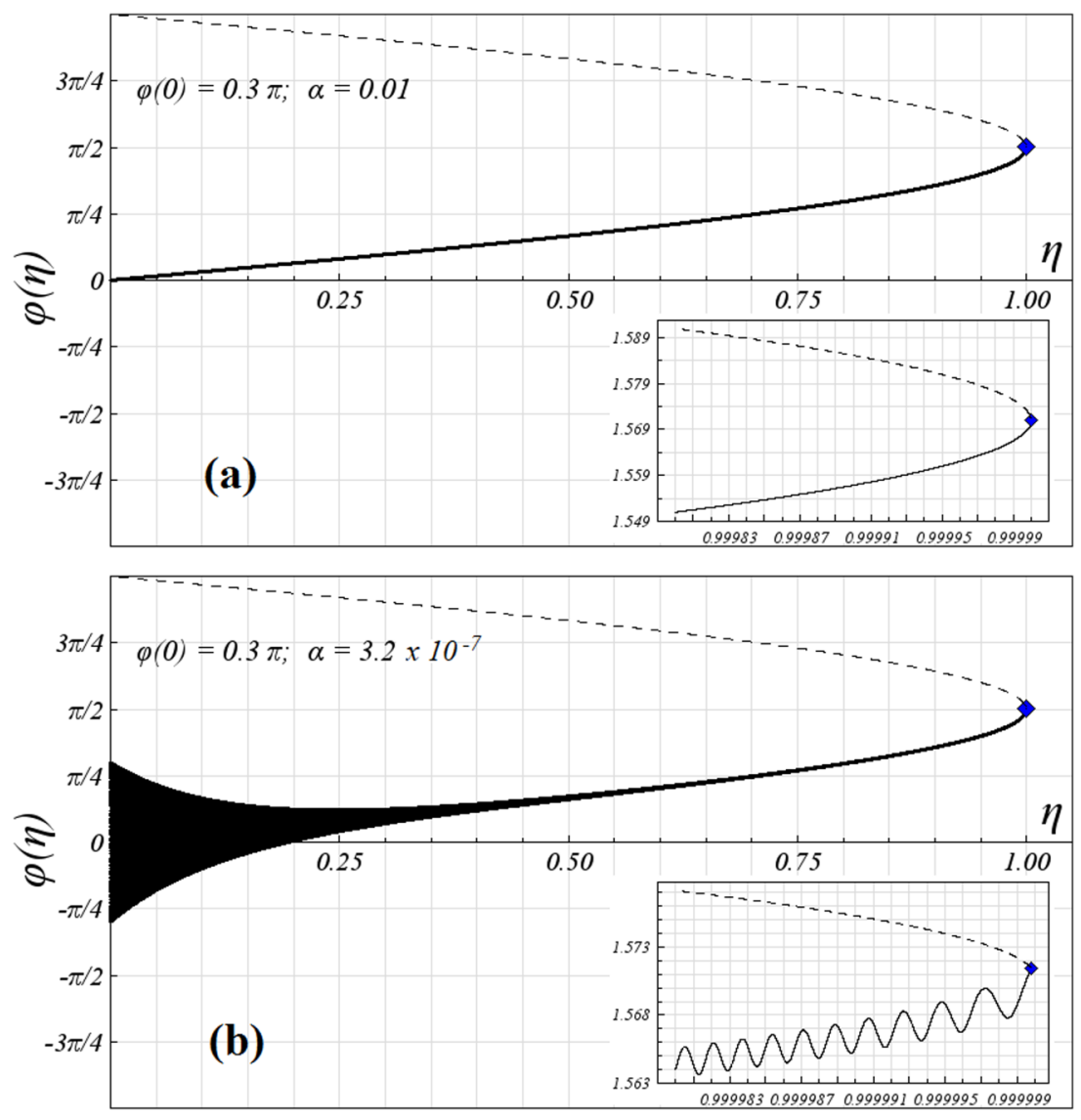

FIG. 3. The changes in stability and equilibrium generated lowering loss, setting as initial conditions on the phase $\varphi_{0}=0.3 \pi, \dot{\varphi}_{0}=0$. We can see in (a) that for $\alpha=0.01$ the response is identical to that of the panels of Fig. 2. However, decreasing the loss $\alpha$ parameter to $3.2 \times 10^{-7}$ in (b), and to $3.2 \times 10^{-8}$ in (c), the $\eta$ point for which the two curves cross becomes less than unity, since the initial oscillations due to the initial angle do not damp out. The fine structure of the dark areas is evident in the magnifying zoom showing the phase oscillations and the crossing with the instability curve.

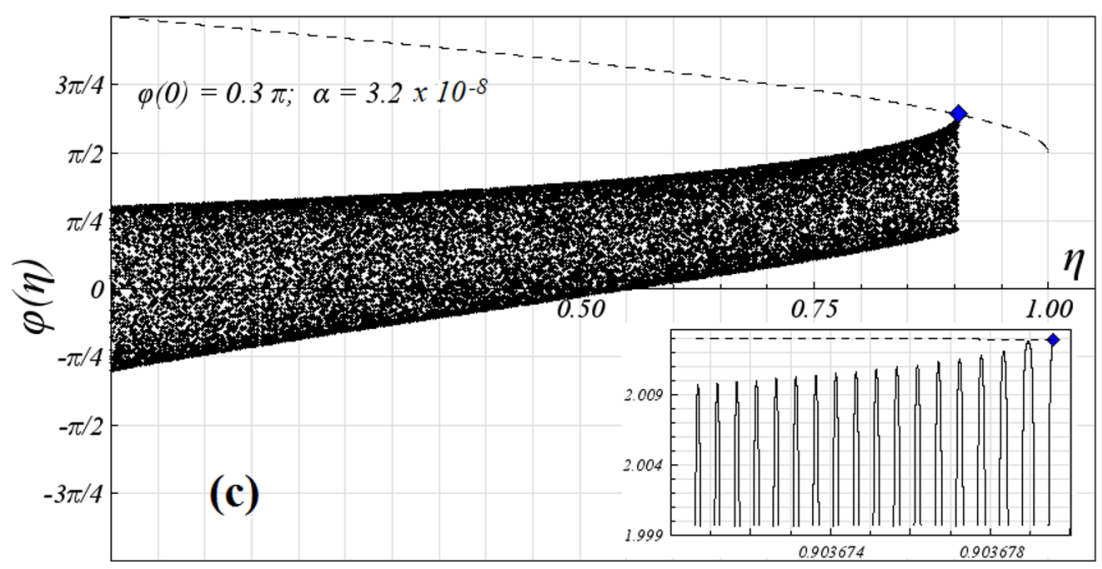

escapes from the potential well for $\eta$ values very close to the critical one (the unity); however, for $\alpha$ in the $10^{-7}$ range, the phase oscillations due to the initial angle do not damp out and the dark area in the figures is just generated by the oscillations whose period is too small to be seen in the figures [we just show a zoom with few periods before escaping in the inset of Fig. 4(b)]. In this case, the escape occurs, as shown in the figure, when the phase oscillation trajectory crosses the unstable point trace for $\eta<1$. Considering the effect shown in Figs. 3 and 4, we decided to investigate systematically the effect of dissipation and initial conditions on the escape current, namely, the value of the bias current for which the system escapes from the well.

The result of our simulations for the dependence of the escape current upon loss parameter and initial angle is epitomized in Fig. 5. Here, we show in a three-dimensional plot the data obtained for the escapes out of the potential well of the system (2) for $T=0$ setting 

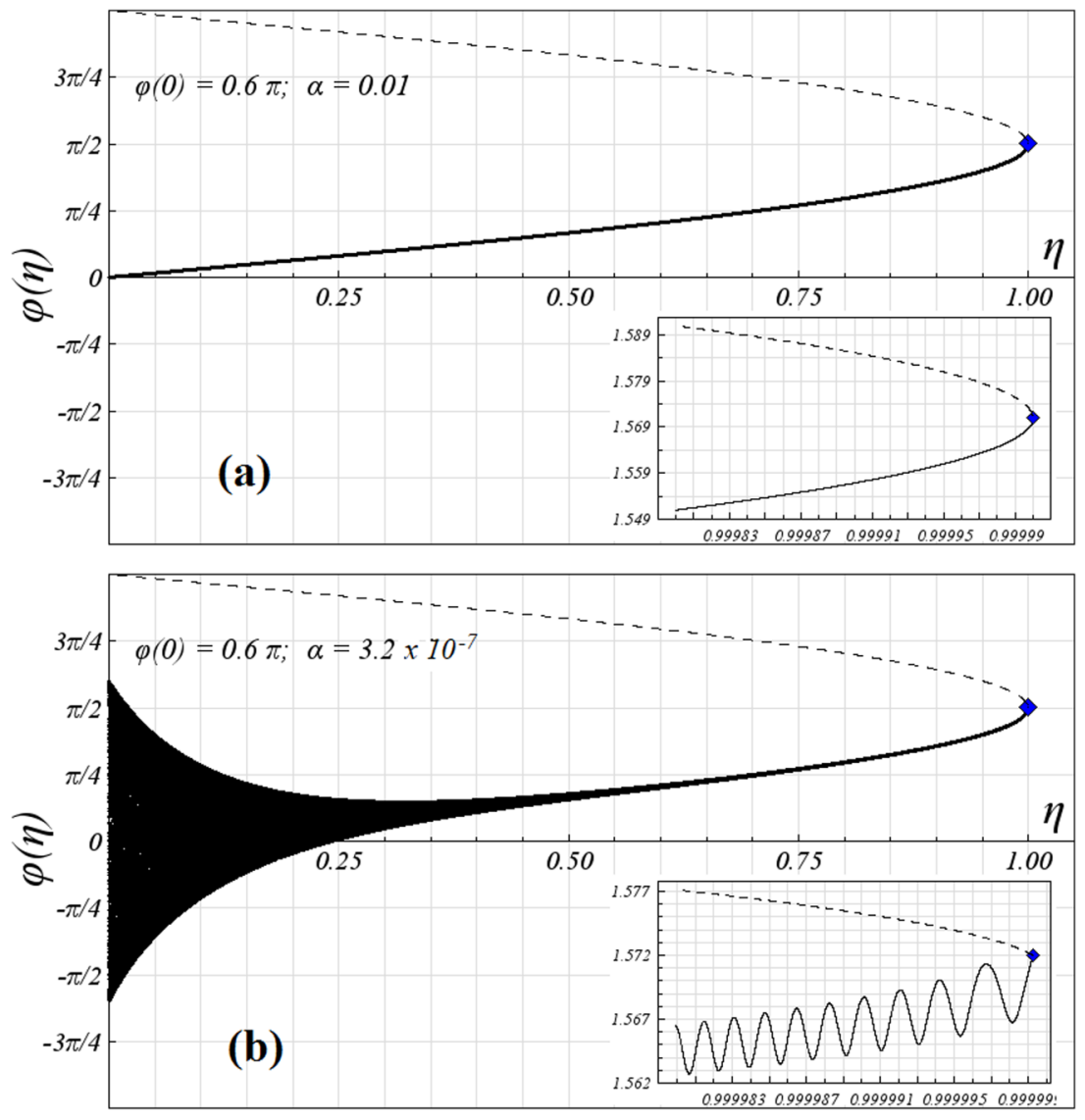

FIG. 4. Same as in Fig. 3, for initial conditions, $\varphi_{0}=0.6 \pi$ and $\dot{\varphi}_{0}=0$. We see now in (b) and (c) that the escape occurs in values of $\eta$ lower than those recorded in Fig. 3.

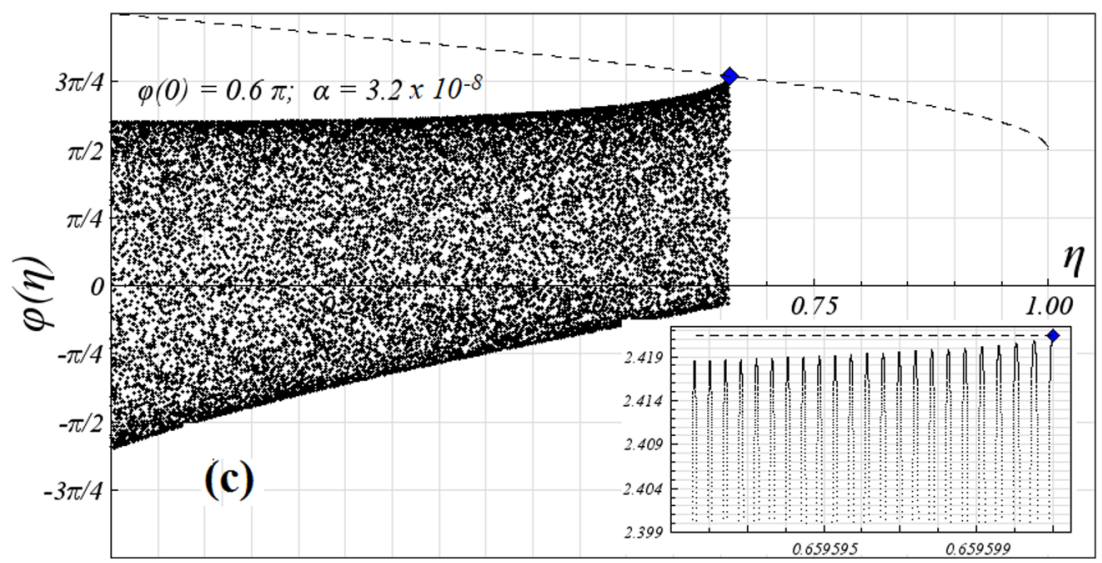

$\dot{\eta}=1.95 \times 10^{-8}$. On the vertical axis, we report the observed value of the "escape" of $\eta_{E}$, namely, the value of $\eta$ generating an escape event from the periodic oscillations in the potential well to an out of the well running state in which the angle increases continuously. On the horizontal axes, we have the initial angle $\varphi_{0}$, which is indicated in the figures as $\varphi_{0}$ and the loss parameter $\alpha$. We can see that when the initial angle tends to zero, the escape always occurs for $\eta_{E}=1$, for any value of the loss parameter. Instead, when the loss decreases and the initial angle increases, we see that the escape current decreases substantially until it reaches zero actually when $\varphi_{0}= \pm \pi$ and values of the loss parameter less than $10^{-7}$. Changing the value of the parameter $\dot{\eta}$ of one order of magnitude, no substantial differences are observed in the escape plots of Fig. 5: we always see that relevant changes occur below $\log \alpha \cong-7$.

In Fig. 6, we show two sections of plots like that of Fig. 5 where we can see more clearly the effect of the loss parameter 


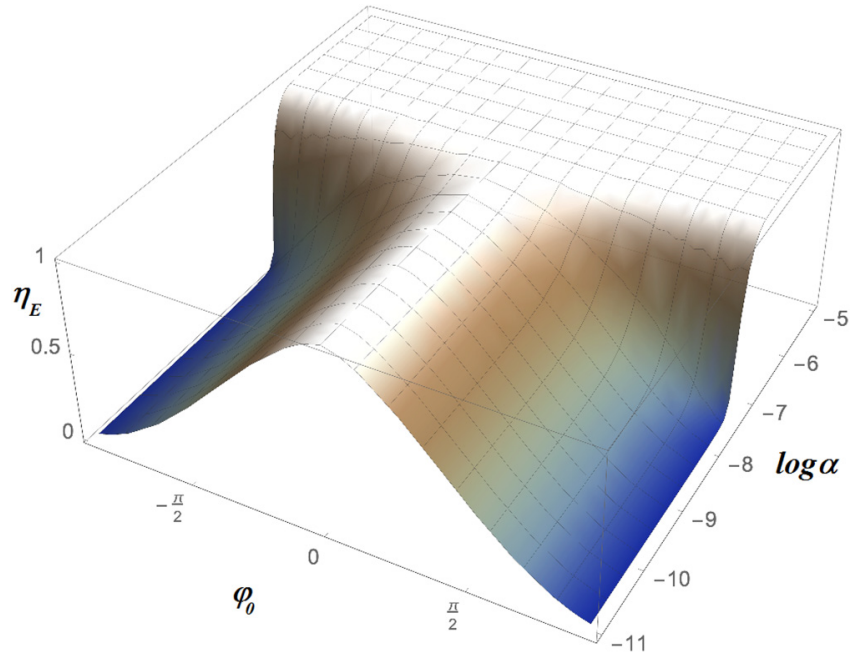

FIG. 5. 3D plot showing the dependence of the "escape" values of $\eta$ upon variations of the loss term and initial angle. The plot is obtained for a sweep rate $\dot{\eta}=1.95 \times 10^{-8}$.

condition (a) and initial conditions (b): these are used as parameters for the curves in the figures where, on the vertical axis, we always report the value of the escape current. Both these "sections" were obtained for $\dot{\eta}=1.95 \times 10^{-8}$. In (a), we can clearly see that the escape is very flat down to $10^{-7}$ and then it becomes a monotonically decreasing and symmetric function of the angle. Decreasing the loss, the width of the bell-shaped function shrinks but saturates for $\log (\alpha)=-9$. In (b), we show the dependence of the escape current upon the parameter $\alpha$ for different initial conditions: here, we plot the escape current vs the $\log \alpha$ and we parametrize with respect to the initial angle, and the result of this operation is just to set the value of a specific escape current on the left side of the plot, while on the right side, for $\alpha>10^{-7}$, the escape current is always equal to the unity.

We then investigated the response of the system by changing the parameter $\dot{\eta}$ for a given initial angle, $\varphi_{0}=0.2 \pi$, and different values of the loss parameter. The results are shown in Fig. 7: here, in (a), we see that variations in $\dot{\eta}$ even of one order of magnitude generate slight differences in the escape currents around the loss values of the order of $10^{-7}$; the interesting thing now is to consider what happens if we scale the horizontal axis of the data in (a) plotting the escape current as a function of the common logarithm of ratio $\kappa=\frac{\alpha}{\dot{\eta}}$. Now we find [see Fig. 7(b)] that all the data collapse in a single curve. The data show that $\kappa$, the ratio between loss parameter $(\alpha)$ and rate of increase of the forcing term $(\dot{\eta})$, plays an important role providing a sort of normalization of the observed potential escape features and a discontinuity in the response of the system occurs when $\kappa=1$. The result of Figs. 2-7 summarize our investigations for $T=0$ : given a specific initial condition on the angle coordinate of the potential, the value of $\eta$ for which we observe escape out of the potential well depends in a relevant way from the values of the parameters $\alpha$ and $\dot{\eta}$.
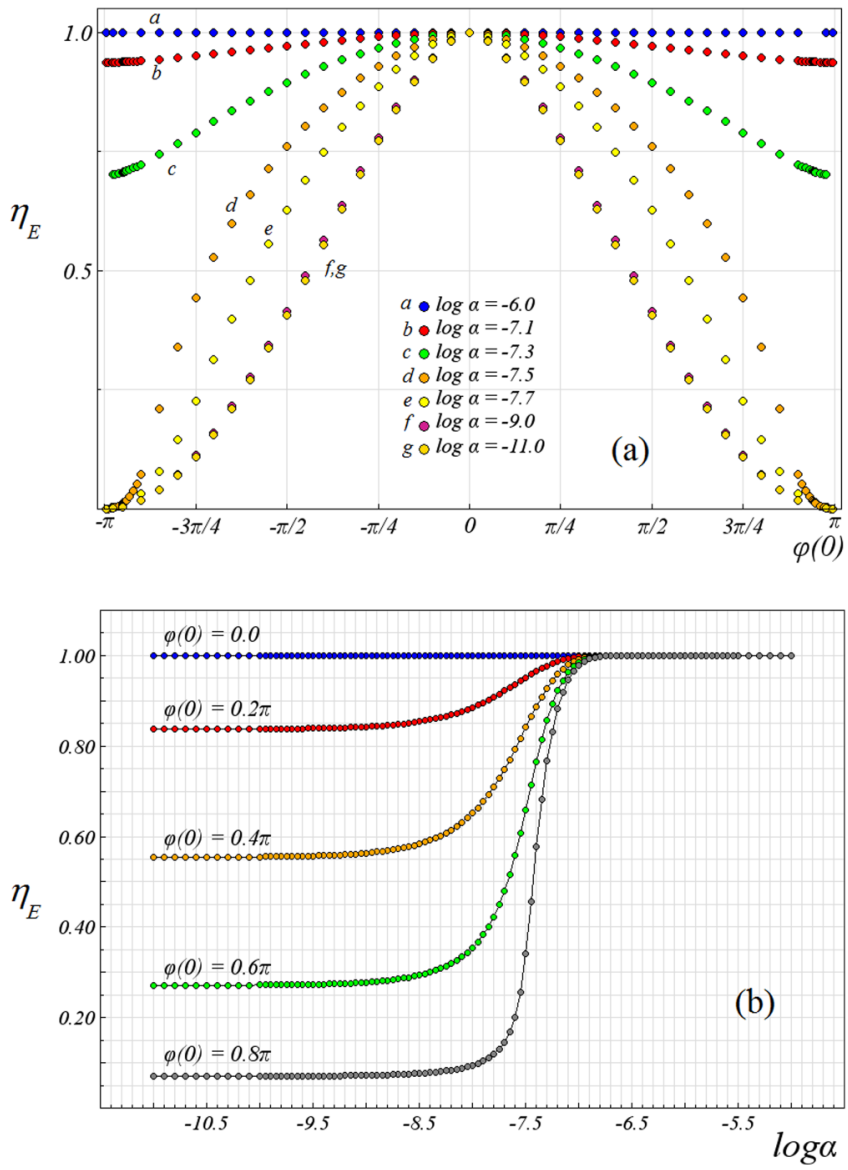

FIG. 6. Projections of the 3D plot of Fig. 5 on the $\left(\eta_{e}, \varphi\right)$ plane (a) and on the $\left(\eta_{e}, \log \alpha\right)$ plane (b).

We conclude that the system (2) undergoes an abrupt "transition" when $\kappa$, the ratio between dissipation and rate of increase of applied force, is equal to the unity. This ratio has also been shown to play a relevant role in the context of the appearance of multipeaked escape statistical distributions when sweep rates are particularly high. ${ }^{14,15}$ Since we limit the investigation herein to statistical distributions obtained with relatively low sweep rates, multipeaked distributions like those reported in Ref. 14 are not observed. As we shall see now, the analysis for $T=0$ of the escape process is a relevant physical background for understanding thermal excitations in the well. This is not surprising indeed because it is known that the characteristics of a nonlinear system for $T=0$ lead to identifying relevant phenomena, ${ }^{7}$ and it has been shown how complex can be the $T=0$ spectrum of "simple" nonlinear systems ${ }^{16}$ and how anharmonic analysis of the potentials can provide account for experimental results. ${ }^{17}$

In terms of Josephson effect physics, the role of the oscillations generated by an initial angle on the escape value $\eta_{E}$ (the current for which the junctions switch from zero to non-zero 

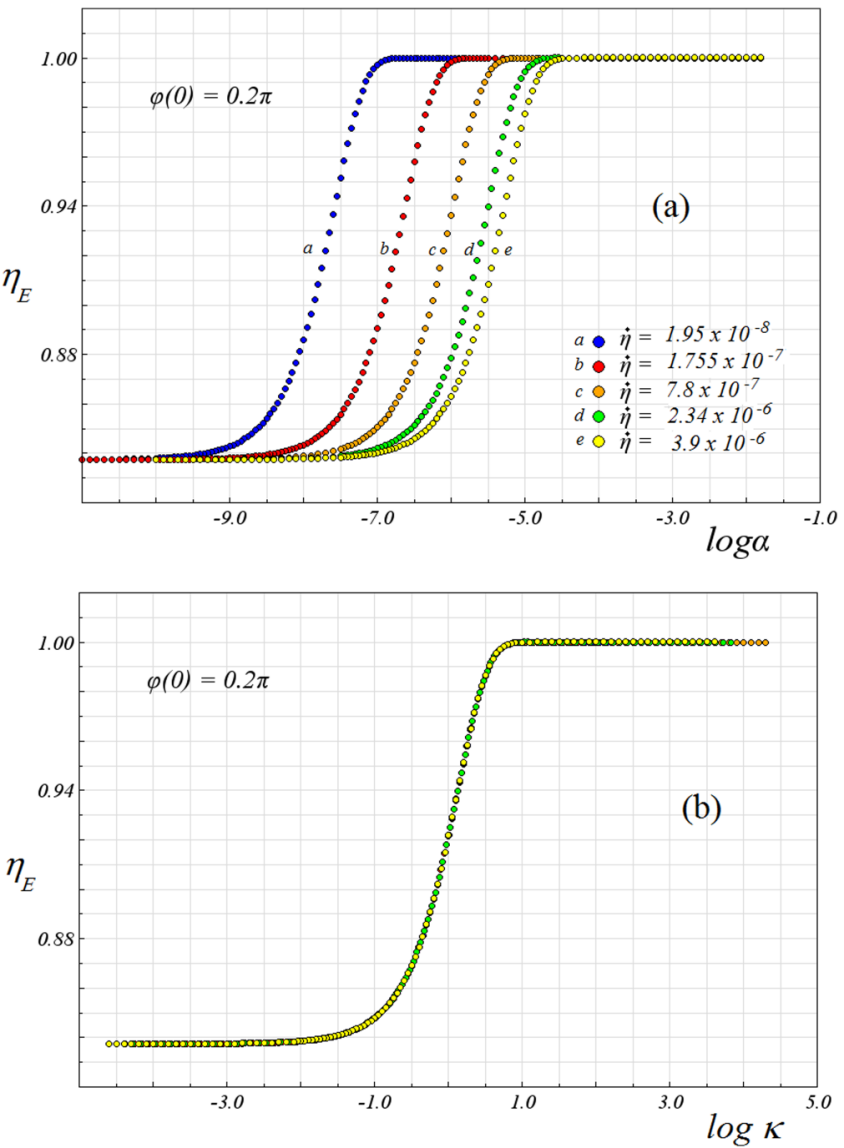

FIG. 7. (a) Dependence of the escape $\eta_{e}$ upon the loss factor $\log \alpha$ with the sweep rate $\dot{\eta}$ set as a parameter; (b) "Normalization" of the data in (a) obtained by scaling the loss factor by the sweep rate. The singularity in the response of the system, as far as escape processes are concerned, occurs when the ratio $\kappa=\frac{\alpha}{\eta}=1$.

voltage state) can be understood, for low dissipation values, on the basis of a model for the energy stored in the Josephson inductance in the zero voltage state. Given the value of the maximum Josephson pairs current $I_{c}$, the Josephson inductance is defined by ${ }^{9}$

$$
L_{J}=\frac{1}{\sqrt{1-\left(\frac{I}{I_{c}}\right)^{2}}} \frac{\Phi_{0}}{2 \pi I_{c}},
$$

where $I$ is the bias current fed through the junction and $\Phi_{0}=2.07 \times 10^{-15} \mathrm{~Wb}$ is the flux quantum. In the limit $I=0$, the above equation returns the zero-bias Josephson inductance $L_{J 0}=\Phi_{0} / 2 \pi I_{c}$.

An initial angle $\varphi_{0}$ gives rise, according to the Josephson dc equation, to an initial current in the form $I_{0}=I_{c} \sin \varphi_{0}$. We assume that, for $t>0$, an oscillating current with amplitude $I_{0}$ and oscillating time dependence in the form $I_{0} \sin \omega_{j} t$ will be activated. Here, we can consider $\omega_{j}$ as the bias-dependent Josephson angular plasma oscillations frequency. ${ }^{9}$ In this harmonic approximation, it is straightforward to calculate the average energy in one period of these oscillations in the Josephson inductor as $W_{L}=\frac{1}{4} L_{j} I_{0}^{2}$.

We presume that, when damping is very low, this initial energy will persist in the system even when the value of the forcing term is slowly increased and indeed these oscillations are those that we can see in the insets of Figs. 3 and 4 . Thus, the just calculated $W_{L}$ will contribute to lower the barrier height $\Delta U$ of the washboard potential in one period in which an escape attempt occurs. We recall now that in the expression for $\Delta U$ given before, in Josephson's terms, energies are normalized to the Josephson zerobias energy, namely, $E_{J}=\Phi_{0} I_{c} / 2 \pi$. Normalizing $W_{L}$ to this quantity and subtracting it from $\Delta U$, we get an effective height of the potential $\Delta U_{E}$ in the form

$$
\Delta U_{E}=2\left(\sqrt{1-\eta^{2}}-\eta \cos ^{-1} \eta\right)-\frac{\eta_{0}^{2}}{4 \sqrt{1-\eta_{0}^{2}}},
$$

where $\eta_{0}=I_{0} / I_{c}=\sin \varphi_{0}$ is the initial, normalized, current amplitude. From this equation, the value of $\eta=I / I_{c}$ for which the escape to the voltage state in a Josephson junction occurs can be easily evaluated as the value $\eta_{E}$, for which $\Delta U_{E}$ goes to zero. A comparison of such a calculation with the numerical data is shown in Fig. 8.

In Figure $8(\mathrm{a})$, we have compared the analytical prediction obtained from (4) with the numerical data for values of $\dot{\eta}$, the current sweep rate in Josephson terms, varied over three orders of magnitude setting $\alpha=0$ (the analytical calculation, in principle, is valid in this limit): we see that the agreement between Eq. (4) and numerical data is very good for $0 \leq \varphi_{0}<1$. In Fig. 8(b) instead we investigate, for a fixed value of the sweep rate, $\dot{\eta}=1.8 \times 10^{-6}$, the effect of different values of the dissipation on the escape current. We see here that the agreement between the predictions and the numerical is very good when $\alpha$ is lower than the sweep rate, the condition in which we observed the relevance of the initial conditions. This confirms the relevance of the parameter $\kappa$ and the physical nature of the phenomenon generating early escapes that are due to the extra energy pumped in the system by the initial condition when $\kappa<1$. In these conditions, we see that the proposed lowering of the potential predicted by Eq. (4) gives a very good prediction of the escape current. Note in Fig. 8(b) that, for a given value of the sweep rate, when the "saturation" value of $\alpha$ is reached (below the normalized sweep rate), further lowering it does not produce relevant changes in the escape current. For high values of the loss parameter, as epitomized in Fig. 5, there are no changes of the escape currents and the escape value always equals 1 .

\section{NON-ZERO TEMPERATURE}

Let us step now to the analysis of the escape process for nonzero temperatures. Thermal fluctuations are plugged into the right hand side of Eq. (2) by the term $n(t)$ linked to the dissipation through the fluctuation-dissipation relationship: ${ }^{18}\langle n(t)\rangle=0$ and $\left\langle n(t) n\left(t^{\prime}\right)\right\rangle=2 \alpha \frac{k_{B} T}{E} \delta\left(t-t^{\prime}\right)$, where $T$ is the thermodynamic temperature of the system, $k_{B}=1.38 \times 10^{-23} \mathrm{~J} / \mathrm{K}$ is Boltzmann's constant, and $E$ is an adequate normalizing energy (in Josephson effect terms, this is just the $E_{J}$ introduced in Sec. II). In Fig. 9(a), 

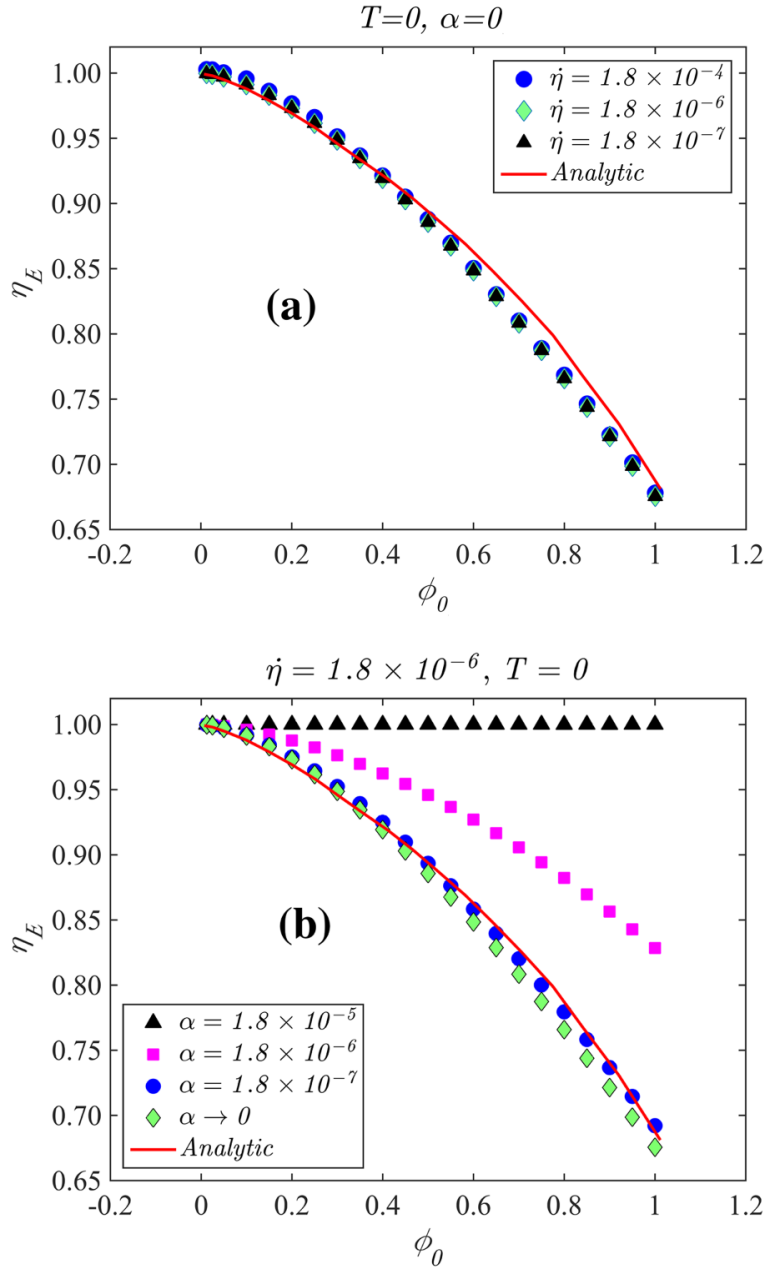

FIG. 8. (a) Comparison between the prediction of Eq. (4) and the numerical simulations for three values of the sweep rate; (b) the results of Eq. (4) compared with numerical data inserting loss in the system. We see that the effect of the internal oscillations due to the initial angle become relevant for $\kappa<1$.

we show three statistical escape distributions obtained for five values of the parameter $\frac{k_{B} T}{E}$, which were, from left to right, respectively, $1.8 \times 10^{-2}, 1.3 \times 10^{-2}, 8.0 \times 0^{-3}, 4.0 \times 10^{-3}, 2.0 \times 10^{-3}$, and $5.0 \times 10^{-4}$. These distributions were obtained by setting the initial conditions $\varphi_{0}=0$ and $\dot{\varphi}_{0}=0$, with standard deviation around these values given by $\left(k_{B} T / E\right)^{1 / 2}$, and setting $\alpha=0.018$ and $\dot{\eta}=1.8 \times 10^{-9}$.

Technically, the distributions of Fig. 9(a) are obtained generating 1500 escape events by increasing $\eta$ and recording each time the value of it for which the escape occurs: on the vertical axis, we just have the number of events (switches) relative to the specific $\eta$ value. The horizontal resolution is $6.4 \times 10^{-5}<\Delta \eta<5.3 \times 10^{-4}$, and it is varied depending on the width of the specific statistical distribution. On the vertical axis, we have essentially a probability
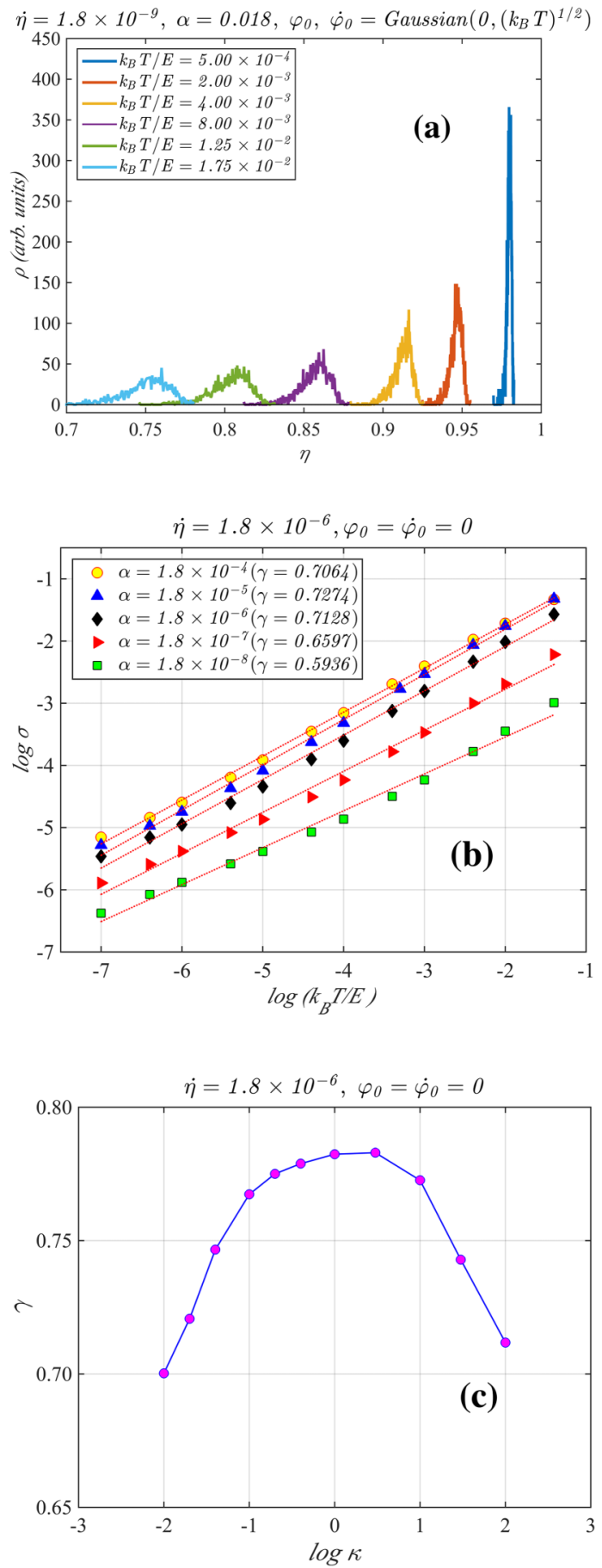

FIG. 9. (a) Typical dependence of the position of the central peak of the statistical escape distribution and of the width of the distribution itself upon temperature: the peaks move toward increasing current and the width of the distributions tends to squeeze; (b) dependence of the width of the distribution statistics upon temperature setting initial angle and phase derivative equal to zero; (c) the dependence of the slopes extracted from the plot (b) upon ratio $\kappa$. 
distribution $\rho$ expressed in arbitrary units. The distributions move right with the temperature, and the width of the statistical distributions decreases with temperature. In Fig. 9(b), we show the temperature dependence of the widths of the statistical distributions for the same value of $\dot{\eta}=1.8 \times 10^{-6}$ but for five different values of the loss factor $\alpha$, which are one order of magnitude apart starting from $1.8 \times 10^{-4}$ down to $1.8 \times 10^{-8}$. We see that the dependencies are straight lines in a log-log plot (here we consider only the data returning linear correlation coefficients above 0.999 ) and the values of the slope return the value of the exponent $\gamma$ of the power law indicated in the inset that varies in the interval $[0.59 ; 0.70]$. The Kramers theory [see Eq. (12) of Ref. 11, the paper is devoted to Josephson phenomenology] predicts a dependence $\sigma \approx\left(k_{B} \cdot T / E_{J}\right)^{2 / 3}$ of the width of the statistical distributions on the temperature; we see that only one straight line of the log-log plot returns a value close to $2 / 3$ while the other values are different up to $10 \%$ from this value.

In Fig. 9(c), we show the dependence of the slope of the straight lines of Fig. 9(b), namely, the exponent $\gamma$ of the law $\sigma=\left(\frac{k_{B} T}{E}\right)^{\gamma}$, upon the parameter $\kappa$. We see that the slope of the curves has a maximum around $\kappa=1$ and is roughly symmetrical around this point. Thus, the dynamical response changes significantly as a function of the ratio $\frac{\alpha}{\dot{\eta}}$ and, since the value of the sweep rate is fixed, the result indicates that is the damping parameter that regulates the response of the system. Analogous results can be obtained varying $\dot{\eta}$.

In Fig. 10(a), we show a plot similar to that of Fig. 9(b) obtained now setting a given angle as initial condition, but still initial angular velocity set to zero, namely, $\varphi_{0}=0.2 \pi$ and $\dot{\varphi}_{0}=0$. For the two lower values of the loss parameter $\left(1.8 \times 10^{-7}\right.$ and $1.8 \times 10^{-6}$ ), we obtain the same value of the exponent of the power law $\gamma$, within few parts over $10^{3}$ uncertainty, and this value is equal to 0.5 . The fitting for the highest loss value instead $\left(1.8 \times 10^{-5}\right)$ returns the exponent 0.672. In Fig. 9(b), we show the dependence of the exponent $\gamma$ of the law $\sigma=\left(\frac{k_{B} T}{E}\right)^{\gamma}$ upon $\log \kappa$. We see here, more clearly than in Fig. 9(c), that the response of the system exhibits abrupt differences below and above $\kappa=1$. It is worth noting that the same dependence shown in Fig. 10(b) is recorded if we set a different angle as the initial condition: along with $\varphi_{0}=0.2 \pi$, we tested the results for and $\varphi_{0}=0.5 \pi$ and $\varphi_{0}=0.1$, and these cases returned exactly the same dependence in the range of $\log \kappa$ shown in Fig. 9(b). We conclude that the effect of setting a given angle as the initial condition is to generate, for $\kappa<1$, a straightforward dependence of the standard deviation of the statistical distributions upon the square root of the temperature $\sigma=\sqrt{\frac{k_{B} T}{E}}$ valid at least over two orders of magnitude of the parameter $\frac{k_{B} T}{E}$.

If we now choose as initial condition an angle distributed randomly and uniformly in a given interval $\left[-\varphi_{0} ; \varphi_{0}\right]$, the above found square root dependence of the standard deviation of the distributions upon $k_{B} T / E$ fails. What happens in this case is quantitatively evident in Figs. 11(a)-11(c) where we show the dependence of the standard deviation of the distributions upon the normalized thermal energy in a log-log plot. Here, we see that for lower values of the normalized energy, a saturation of the standard deviation occurs and the specific saturation current depends on the initial
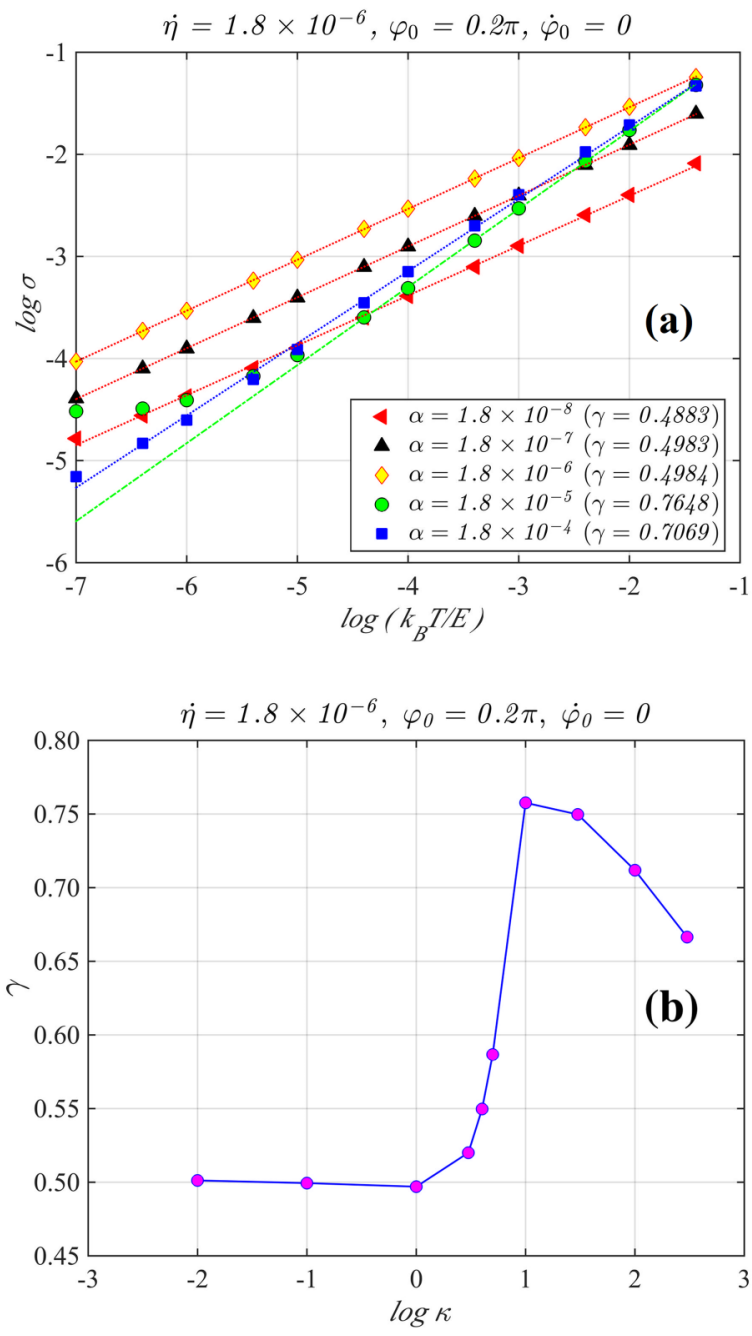

FIG. 10. (a) The dependence of the width of the statistical distributions upon the temperature for non-zero initial angles. The values of the initial angles are indicated in the plot. In (b), we see the dependence of the slope upon the parameter $\kappa$ when the initial angle is fixed to $0.2 \pi$. We can clearly see that passing through the interval $0<\kappa<1$, the response of the system strongly changes.

angle. The result are obtained setting an initial angle randomly uniformly distributed respectively in the intervals $[-0.1 ; 0.1]$ (a), $[-0.2 \pi ; 0.2 \pi](b)$, and $[-\pi / 2 ; \pi / 2]$ (c). In the plots, we also show the dependence of the "saturation" curves upon the loss parameter. Looking back at the $T=0$ results, we can have a straightforward explanation of this effect considering, in particular, Fig. 6(b). We see there that different initial angles correspond to different escape currents and, therefore, if the initial angle is uniformly distributed over an interval, the escape $\eta_{E}$ also shall be distributed over an interval that will set a distribution even for $T=0$. When the temperature in the system is high enough, these initial conditiongenerated oscillations shall not be visible because they are masked 

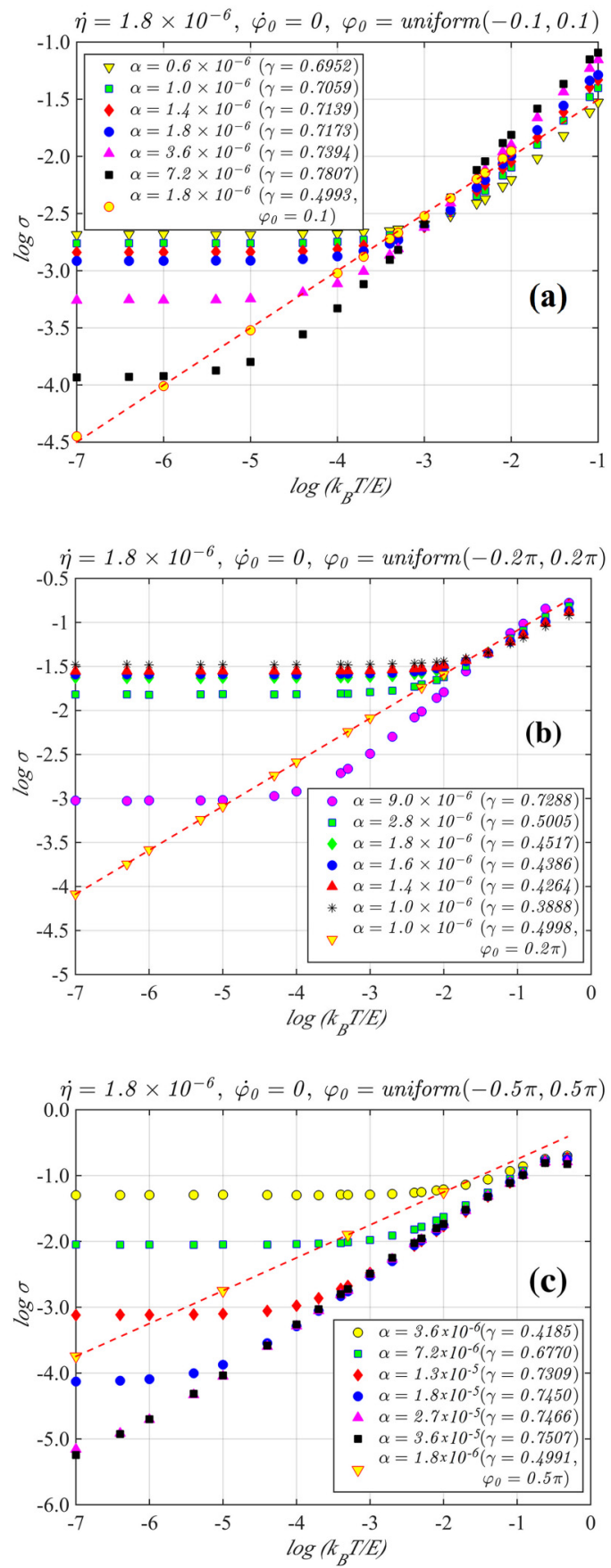

FIG. 11. The saturation of the widths of the distributions for different values of the initial angle distribution. In (a), (b), and (c), we set as initial condition and angle uniformly distributed respectively in $[-01 ; 0.1],[-2 \pi ; 2 \pi]$, and $[-5 \pi ; 5 \pi]$. In each plot, the different symbols indicate different values of the loss parameter indicated in the inset. In the insets, we also indicate the "asymptotic" high-temperature value of the exponent $(\gamma)$ of the law $\sigma=\left(k_{B} T / H_{j}\right)^{\gamma}$. The dotted straight lines indicate, for comparison, the behavior observed for a fixed angle: in this case, we observe no saturation all over the investigated temperature range. by thermal fluctuations; however, when thermal fluctuations have a low energy, the standard deviation is just determined by the randomness of the initial angle distribution.

In Figs. 11(a)-11(c), we also write the slopes of the "linear" portions of the plots, those corresponding to higher temperatures. It is worth noting that results similar to those of Fig. 10, obtained setting an initial uniform random distribution of the angle, can be obtained even with different types of random initial conditions.

The above argument concerning the saturation of the standard deviation of the escape distributions based on the $T=0$ behavior, makes sense when $\kappa$ is of the order of the unity and below. When $\kappa$ is much above the unity, the distribution of escape values does not depend on the initial angle, as we see in Figs (3)-(7), and, therefore, saturation cannot be expected on the basis of the above conjecture. However, in the nonzero temperature case, variations of the plots of Figs. (3)-(7) can be expected and saturation effects are recorded even at very low temperatures even for $\kappa>1$, as shown in Fig. 11. We see in this figure that the "saturation" $\sigma$-value of statistical distribution at low energies/temperatures depends on the amplitude of the random initial angle interval and on the value of the loss parameter.

We have seen in Fig. 11 that, for a given value of $\dot{\eta}$, the width of the distributions can attain a saturation value that will call $\sigma_{S A T}$. In Fig. 12, we show the dependence of this parameter upon the loss parameter $\alpha$ in a semi-logarithmic plot for three settings of the initial conditions corresponding to have the angle uniformly distributed respectively in $[-0.1 ; 0.1],[-0.2 \pi ; 0.2 \pi]$, and $[-0.5 \pi ; 0.5 \pi]$ : Fig. 12(a) has been obtained for $\dot{\eta}=1.8 \times 10^{-6}$. Instead, in Fig. 12(b), we show the same dependencies of the saturation currents, for the same initial conditions, obtained for $\dot{\eta}=1.8 \times 10^{-7}$. We clearly see in both Figs. 12(a) and 12(b) that the dependencies are exponential of the type $\sigma_{S A T}=\sigma_{0} e^{-s \alpha}$ with slopes $s$, indicated in the insets, which have only a few percent differences between the two cases (for the same random initial interval). For both cases, we see that the extrapolation of the straight lines toward $\alpha=0$ is a well defined, non-zero, value and, therefore, for a random initial angle, the width of the distribution has a finite value in a lossless system. "Parental" results of those shown in Figs. 12(a) and 12(b) were recently obtained in Ref. 14 for the width of the peaks appearing in the statistical distributions for a high sweep rate: in that case, the dependence of the width of the individual peaks of a multipeaked distribution was investigated, and it was shown that the dependence of the width of the peaks on the loss factor was exponential.

\section{EXPERIMENTAL BACKGROUND}

We note that the work herein contained is referred to very adiabatic changes of force terms in system (2). The effects of "pulsed" excitations on this system have been thoroughly investigated in previous publications (see Ref. 10 and references therein). The pulsed experiments on our systems and the oscillations these generated are much reminiscent of the phenomenology early observed in pulsed laser experiments; ${ }^{19}$ in the present paper, however, we have undertaken a systematic analysis of the "adiabatically" driven system (2) since we believe this effect can serve as a useful background of knowledge for investigating more complex and non-adiabatic 

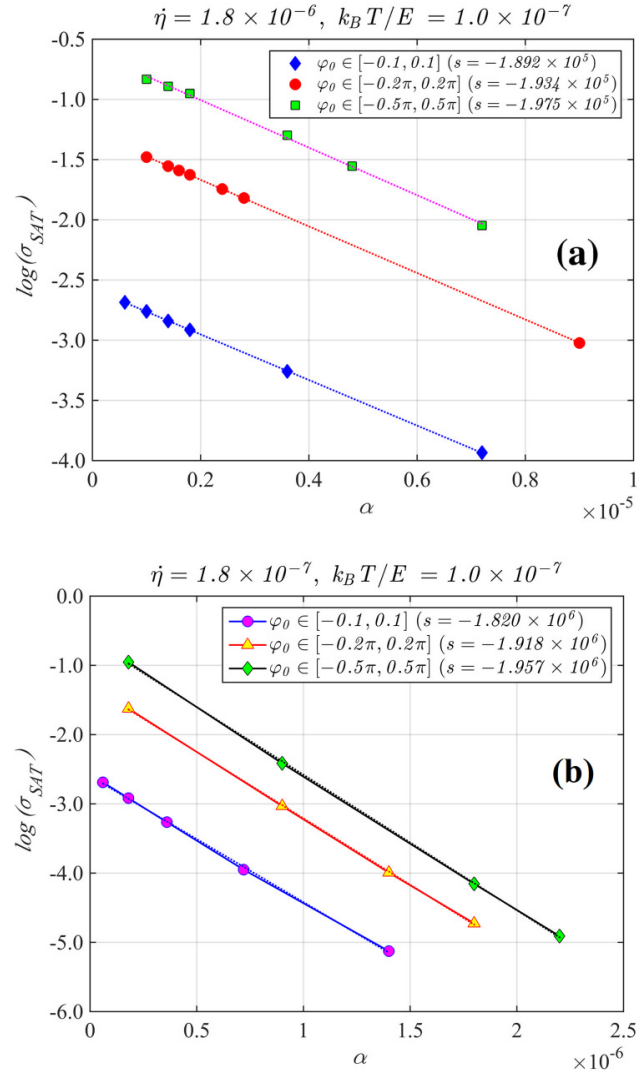

FIG. 12. The dependence of the value of the distribution width saturation value $\sigma_{S A T}$ upon the loss parameter for different initial conditions. In the semi-log plot, we can clearly see an exponential dependence of the saturation width upon the dissipation, and in the insets, we indicate the slope of the linear dependence. In (a), we have set a rate of increase of the force term $\dot{\eta}=1.8 \times 10^{-6}$ while in (b) $\dot{\eta}=1.8 \times 10^{-7}$.

conditions. This was the case indeed of the work recently appeared in Ref. 14 for which a guide from (preliminary) results herein presented was important.

Framing of our results in terms of experimental parameters and procedures can be illustrated for a specific case. Systems that have represented a benchmark for the one-dimensional potential herein treated are the Josephson junction and interferometers (consisting of a superconductive loop closed by one or two junctions). The first thing to point out is that the operating temperatures at which Josephson systems have been typically operating have gone from $4.2 \mathrm{~K}$ down to $10 \mathrm{mK}$. What we have used as normalizing energy $E$ is, in Josephson terms, the zero-bias Josephson energy, which is the $E_{J}$ defined earlier in Sec. II. Now, typical maximum pair currents for the thermal activation experiments performed on Josephson junctions are in the range $(1-330) \mu \mathrm{A} .^{20-26}$ If we consider, for example, a $1 \mu \mathrm{A}$ Josephson current, the corresponding Josephson energy is $E_{J}=0.33 \times 10^{-21} \mathrm{~J}$ and our normalized $k_{B} T / E_{J}$ will be 0.16 at $4 \mathrm{~K}$ and $4 \times 10^{-4}$ at $10 \mathrm{mK}$. When the Josephson maximum current scales up one order of magnitude, the energy will scale up the same amount and the maximum and minimum values of the normalized energy will consequently scale down by a factor 10. Thus, for a Josephson current of $100 \mu \mathrm{A}$, the energy $E_{J}=33 \times 10^{-21} \mathrm{~J}$ and the normalized temperature will range from $0.16 \times 10^{-2}$ at $4 \mathrm{~K}$ down to $4 \times 10^{-6}$ at $10 \mathrm{mK}$.

From the above estimate of currents and related normalized energy, it is evident why we have chosen the intervals of energy variation for our investigation. Other relevant parameters in our work are the rate of change of the applied force $\dot{\eta}$ and the loss factor $\alpha$; as far as the first parameter is concerned, also known in Josephson terms as normalized sweep rate since it is the normalized time derivative of the bias current, this can vary from the range of $10^{-6}$ down to the range of $10^{-9}$, depending on junction properties (essentially capacitance and critical current). ${ }^{20-25} \mathrm{~A}$ special problem instead is represented by establishing the effective losses in Josephson systems when performing potential escape measurements; in this case, the work reported in Ref. 27, for example, provides interesting information because it evaluates a specific temperature dependence of the dissipation in the junctions and simulations should take into account this physical condition. An analysis of the intriguing effects generated by the variations of this parameter would be interesting and requires further investigations.

In the present work, we have preferred to set constant the dissipation parameter $\alpha$ in order to maintain our analysis more general because in many physical cases the dissipation coefficient can just be kept constant. As we have seen at the beginning setting a constant dissipation factor and all zero initial conditions, there are no effects on the escape from the potential and the data indeed might follow very accurately the Kramers model. In Fig. 13, we plot the numerically obtained position (in current) of the statistical distribution peaks with initial phase and phase derivative set to zero, for $\alpha=0.01$. The full circles represent the numerical results for the position in current of the statistical peak of the distributions, in values normalized to $I_{c}$ while the continuous line represents the prediction of Kramers theory. ${ }^{28}$ According to this theory, the position, in current $\left(I_{p}\right)$, of the peak of the statistical distribution moves away from the $T=0$ Josephson critical current $I_{c}$ following the same functional dependence on the temperature of the width of the distribution, namely, $\left(I_{c}-\left\langle I_{p}\right\rangle\right) / I_{c} \approx\left(k_{B} T / E_{j}\right)^{2 / 3}$. A numerical evidence of the fact that peak widths and the position follow the same temperature dependence is given in Fig. 5(1), right panel, of Ref. 10: as we see there, for the given value of the loss, the data follow perfectly Kramers predictions all over the temperature range.

However, if the loss factor $\alpha$ is scaled down to $10^{-10}$, namely, below $\dot{\eta}=2 \times 10^{-9}$, so that the parameter $\kappa=\frac{\alpha}{\dot{\eta}}$ is below the unity; at low temperatures, the position of the peaks freezes as shown by the yellow triangles in Fig. 13. For this specific run, we have chosen to set "Gaussian" initial conditions meaning that the initial angle is randomly distributed around the given angle $\varphi_{0}$ $=0.0856 \pi$ with a standard deviation of the packet proportional to the square root of the $k_{B} T$; the initial phase derivative is always set to zero. The triangles, squares, and diamonds in Fig. 13 correspond to the time steps of the numerical integration indicated in the inset, showing stability of the results, for very low dissipation, under "halving" tests. The intersection of horizontal line obtained for 


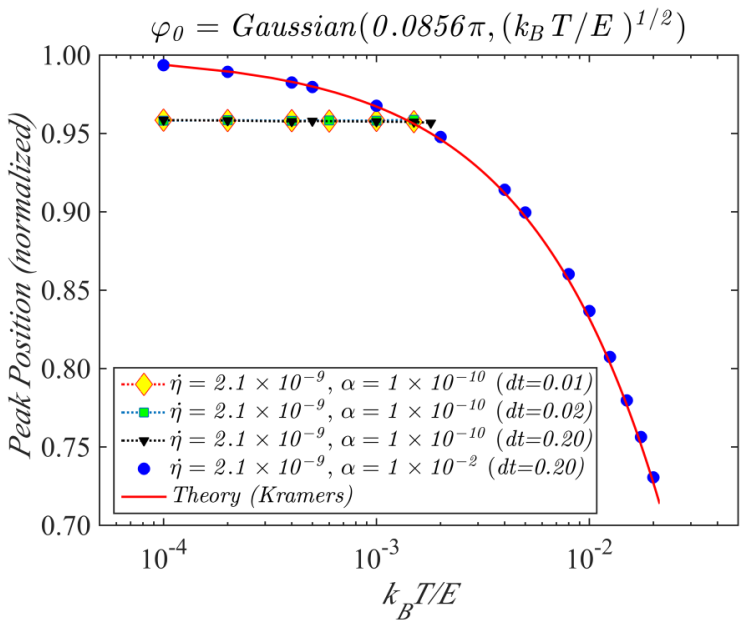

FIG. 13. The dependence of the current corresponding to the peak of the statistical distributions upon temperature as described by the Kramers model (continuous line) and the results of numerical simulations (blue full circles) obtained setting all zero initial conditions, $\alpha=0.01$ and $\dot{\eta}=2.085 \times 10^{-9}$. The yellow triangles, the squares, and the diamonds show the results obtained, at low temperature, setting $\alpha=10^{-10}$ the same value of $\dot{\eta}$ but initial conditions set by $\varphi_{0}$ $=0.0856 \pi$ (with Gaussian noise around it) and $\dot{\varphi}(0)=0$. The $d t$ in the inset are the values of the time steps set for performing halving checks for the numerical integration.

$\alpha=10^{-10}$ with the Kramers curve occurs for a temperature slightly above $50 \mathrm{mK}$. We can say that an initial angle $\varphi_{0}=0.086$ and a loss factor less than the normalized sweep rate establish a difference between the temperature intervals in which the system responds according to the Kramers model or to the phenomenology of a very underdamped dynamical regime. As attempted frequency for tracing the theoretical Kramers curve, based on Eq. (1), in Fig. 13, we have used both the one corresponding to the harmonic approximation, $f=f_{J 0}\left(1-\eta^{2}\right)^{1 / 4}$ (where $f_{J 0}=\frac{\omega_{0}}{2 \pi}$ is the zero-bias Josephson plasma frequency ${ }^{9,10}$ ) and the one coming from the anharmonic approximation (see page 5 of Ref. 10), but the two results are identical within $0.1 \%$.

We note that, according to the analysis performed in Ref. 27, in a temperature interval between $1 \mathrm{~K}$ and $5 \mathrm{~K}$, the effective damping can change of 5 orders of magnitude, and, therefore, it is not unreasonable to expect that in the hundreds of millikelvin range, the normalized damping could be of the same order of magnitude, or less, of the sweep rate and generate the freezing of the position peaks. Since it has been shown that problems might exist $^{29,30}$ in attributing to quantum phenomenology and theories in experimental results at very low temperatures, we speculate that more a quantitative insight into the Josephson potential escape phenomenology could come from specific analysis of the resistively and capacitively junction model (RCSJ) of Josephson systems considering adequate settings for the effective losses and initial conditions. We note that in very underdamped conditions, oscillating transients can hardly be removed, even imposing waiting times between current ramps.

\section{CONCLUSIONS}

We have investigated systematically the features of potential escape for a physical system having relevant impact in general physics and condensed matter. Although work has been dedicated in the past to identify analytical criteria to describe the response of the system that we have investigated and peculiar models have been considered to explain experiments, ${ }^{20-26}$ the nonlinearity of the potential and the related dynamical equation it generates are such that only accurate and systematic numerical integrations can enable us to have ideas of the response over wide parameters excursions at very low temperatures. The zero temperature analysis of system (2) led us to identify a relevant parameter, the ratio between loss factor and rate of increase of the applied force: a threshold related to it $\left(\kappa=\frac{\alpha}{\dot{\eta}}=1\right)$ characterizes the response of the dynamical system. This threshold differentiates the dynamical response both in zero temperature and in non-zero temperature. Below this threshold, the dynamics is very dependent on the initial conditions imposed on the dynamical variable of the potential because the oscillations generated by the initial data condition the potential escape process. Moreover, we have shown that relevant deviations from the predictions of the Kramers model occur due to the setting of specific random initial data.

The present paper was also motivated by the fact that in a previous publication, ${ }^{30}$ evidence was found that macroscopic quantum tunneling theory cannot provide explanation of experimental results obtained on Josephson systems at a very low temperature. We have herein shown that specific settings of the RCSJ model, as far as the Josephson phenomenology is concerned, ${ }^{9,10}$ can return results that are close to the observed experimental phenomenology in terms of saturation of the statistical distributions and freezing of the position of the peak of the distributions. The physical motivation providing agreement between modeling and experiments is essentially the fact that in rather underdamped classical systems, the effects of transient oscillations cannot be neglected.

The results herein presented are consistent with recently reported investigations of potential escape phenomena in which the effect of very high rates of change of the external force on system (2), with the noise term, was investigated. ${ }^{14}$ In Ref. 14, it was shown that the threshold $\kappa=1$ represents a crucial condition for generating multipeaked statistical distributions. It is evident then that the behavior of the system for $T=0$ is a background conditioning the response when thermal excitations are present. It will be surely interesting to further develop specific issues related to the Josephson effect and, in particular, those concerning the temperature dependence of the dissipation parameter. Previous works indeed have attempted to find general criteria for the appearance of specific phenomena in the escape process. ${ }^{31}$

It is also worth noting that, presently, much attention is devoted to digital circuits based on the Josephson effect, which could work without the shunting resistors necessary for the proper operation of Rapid Single Flux Quantum (RSFQ) logics. ${ }^{32-34}$ In these conditions, in which the effect of loss is pushed to an extremely low limit, the response of the Josephson junctions to external excitations leading it to fast switches is a crucial phenomenon and the effect of the initial state of the system is even more 
relevant than it is for shunted junctions. ${ }^{35}$ The same type of problems are faced when considering unshunted Josephson junctions as switches for single photon detectors, ${ }^{26,36,37}$ a field which has a promising impact both on fundamental and applied sciences. The relevance of our work can be particularly realized when considering the issues in Ref. 26. It is important in this specific case to set the optimal parameters for which the response of the devices is not conditioned by internal modes or spurious oscillations.

\section{ACKNOWLEDGMENTS} (Italy)

This work was partially supported by the INFN-FEEL project

\section{REFERENCES}

${ }^{1}$ A. Politi, in Stochastic Fluctuations in Deterministic Systems in Large Deviations in Physics, Lecture Notes in Physics Vol. 885, edited by A. Vulpiani, F. Cecconi, M. Cencini, A. Puglisi, and D. Vergni (Springer, Berlin, 2014).

${ }^{2}$ P. Hänggi, P. Talkner, and M. Burkovek, Rev. Mod. Phys. 62, 251 (1990).

${ }^{3}$ A. M. Selvam, Noise or Random Fluctuations in Physical Systems: A Review in Self-Organized Criticality and Predictability in Atmospheric Flows, Springer Atmospheric Sciences (Springer, Cham, 2017).

${ }^{4}$ H. A. Kramers, Physica 7, 284 (1940).

${ }^{5}$ Activated Barrier Crossing, Applications in Physics, Chemistry and Biology, edited by G. R. Fleming and P. Hänggi (World Scientific Eds, Singapore, 1993).

${ }^{6}$ G. L. Baker and J. A. Blackburn, The Pendulum: A Case Study in Physics (Oxford University Press, 2005).

${ }^{7}$ R. L. Kautz, Chaos: The Science of Predictable Random Motion (Oxford University Press, 2011).

${ }^{8}$ P. W. Anderson, "Special effects in superconductivity," in Lectures on the Many Body Problem, edited by E. R. Caianiello (Academic Press, New York, 1964), Vol. 2, pp. 113-135.

${ }^{9}$ A. Barone and G. Paternò, Physics and Applications of the Josephson Effect (John Wiley, New York, 1982); T. Van Duzer and C. W. Turner, Principles of Superconductive Devices and Circuits (Prentice-Hall, Englewood Cliffs, NJ, 1999).

${ }^{10}$ J. A. Blackburn, M. Cirillo, and N. Grønbech-Jensen, Phys. Rep. 611, 1 (2016).

${ }^{11}$ J. Kurkijärvi, Phys. Rev. B 6, 832 (1972).

${ }^{12}$ M. Büttiker, E. P. Harris, and R. Landauer, Phys. Rev. B 28, 1268 (1983).

${ }^{13}$ N. Grønbech-Jensen and O. Farago, Mol. Phys. 111, 983 (2013).

${ }^{14}$ C. Cheng, M. Cirillo, G. Salina, and N. Grønbech-Jensen, Phys. Rev. E 98, 012140 (2018).
${ }^{15}$ N. Grønbech-Jensen and M. Cirillo, Phys. Rev. B 70, 214507 (2004).

${ }^{16}$ J. A. Blackburn, M. Cirillo, and N. Grønbech-Jensen, Eur. Phys. Lett. 115, 50004 (2016).

${ }^{17}$ N. Grønbech-Jensen, M. G. Castellano, F. Chiarello, M. Cirillo, C. Cosmelli, V. Merlo, R. Russo, and G. Torrioli, "Anomalous thermal escape in Josephson systems perturbed by microwaves," in Quantum Computing in Solid State Systems, edited by B. Ruggiero, P. Delsing, C. Granata, Y. Pashkin, and P. Silvestrini (Springer Science, 2006), pp. 111-119.

${ }^{18}$ G. Parisi, Statistical Field Theory (Addison-Wesley, Reading, MA, 1988).

${ }^{19}$ F. T. Arecchi and V. Degiorgio, Phys. Rev. A 3, 1108 (1971).

${ }^{20}$ R. F. Voss and R. A. Webb, Phys. Rev. Lett. 47, 265 (1981).

${ }^{21}$ S. Washburn, R. A. Webb, R. F. Voss, and S. M. Faris, Phys. Rev. Lett. 54, 2712 (1985).

${ }^{22}$ A. Wallraff, A. Lukashenko, C. Coqui, A. Kemp, T. Duty, and A. V. Ustinov, Rev. Sci. Instrum. 74, 3740 (2003).

${ }^{23}$ T. Bauch, F. Lombardi, F. Tafuri, A. Barone, G. Rotoli, P. Delsing, and T. Claeson, Phys. Rev. Lett. 94, 087003 (2005).

${ }^{\mathbf{2 4}}$ K. Inomata, S. Sato, K. Nakajima, A. Tanaka, Y. Takano, H. B. Wang, M. Nagao, H. Hatano, and S. Kawabata, Phys. Rev. Lett. 95, 107005 (2005).

${ }^{\mathbf{2 5}}$ H. F. Yu et al., Phys. Rev. B 81, 144518 (2010).

${ }^{\mathbf{2 6}}$ G. Oelsner, L. S. Revin, E. Il'ichev, A. L. Pankratov, H.-G. Meyer, L. Grönberg, J. Hassel, and L. S. Kuzmin, Appl. Phys. Lett. 103, 142605 (2013).

${ }^{27}$ P. Silvestrini, S. Pagano, R. Cristiano, O. Liengme, and K. E. Gray, Phys. Rev. Lett. 60, 844 (1988).

${ }^{\mathbf{2 8}} \mathrm{K}$. K. Likharev, Dynamics of Josephson Junctions and Circuits (Gordon and Breach, New York, 1986); see Sec. III (eq. 3.65).

${ }^{29}$ J. A. Blackburn, M. Cirillo, and N. Grønbech-Jensen, Eur. Lett. 107, 67001 (2014).

${ }^{30}$ J. A. Blackburn, M. Cirillo, and N. Grønbech-Jensen, J. Appl. Phys. 122, 133904 (2017).

${ }^{31}$ A. L. Pankratov and M. Salerno, Phys. Lett. A 273, 162 (2000); Phys. Rev. E 61, 1206 (2000).

${ }^{32}$ J. Ren and V. K. Semenov, IEEE Trans. Appl. Supercond. 21, 780 (2011).

${ }^{33}$ M. Lucci, J. Ren, S. Sarwana, I. Ottaviani, M. Cirillo, D. Badoni, and G. Salina, IEEE Trans. Appl. Supercond. 26, 100905 (2016).

${ }^{34}$ O. A. Mukhanov, IEEE Trans. Appl. Supercond. 21, 760 (2011).

${ }^{35}$ A. V. Gordeeva and A. L. Pankratov, Appl. Phys. Lett. 88, 022505 (2006).

${ }^{36}$ G. Oelsner, C. K. Andersen, M. Rehák, M. Schmelz, S. Anders, M. Grajcar, U. Hübner, K. Mølmer, and E. Il'ichev, Phys. Rev. Appl. 7, 014012 (2017).

${ }^{37}$ L. S. Kuzmin, A. S. Sobolev, C. Gatti, D. Di Gioacchino, N. Crescini, A. Gordeeva, and E. Il'ichev, IEEE Trans. Appl. Supercond. 28(7), 2400505 (2018). 